\title{
Implementation and outcomes of the RAPID programme: Addressing the front end of the lung cancer pathway in Manchester
}

\author{
Authors: Matthew Evison, ${ }^{\mathrm{A}}$ Kath Hewitt, ${ }^{\mathrm{B}}$ Judith Lyons, ${ }^{\mathrm{A}}$ Phil Crosbie, ${ }^{\mathrm{A}}$ Haval Balata, ${ }^{\mathrm{A}}$ Caroline Gee, ${ }^{\mathrm{C}}$ \\ Rebecca Duerden, ${ }^{\mathrm{D}}$ Melanie Greaves, ${ }^{\mathrm{D}}$ Anna Sharman ${ }^{\mathrm{D}}$ and Richard Booton ${ }^{\mathrm{A}}$
}

\section{Introduction \\ Patients with suspected lung cancer require computed tomography (CT), specialist interpretation of the CT and a consultation with a specialist. Significant time savings could be made with rapid access to these components in the front end of the lung cancer pathway.}

\section{Methods}

The RAPID programme was launched at Manchester's Wythenshawe Hospital in April 2016. This pathway offers next working day $\mathrm{CT}$ for patients with suspected lung cancer, immediate 'hot' reporting of CT images and a same day consultation with a diagnostic specialist.

Results

From April 2016 to January 2019, 1,027 patients were referred to the RAPID programme. The median time from referral to CT was 3 days. The CT was hot reported in $94 \%$ of patients. The median time from CT to triage and consultation with a diagnostic specialist was 0 days. Overall $56 \%$ and $90 \%$ of patients had completed a CT and consultation within 3 and 7 days of referral, respectively ( $0 \%$ and $24 \%$ prior to implementation).

Conclusion

Through simple reorganisation of workload, we have significantly reduced the pathway for patients with suspected lung cancer to meet a specialist with a reported $\mathrm{CT}$, something we firmly believe is replicable across all hospitals.

KEYWORDS: Lung cancer, RAPID programme, pathway

DOI: 10.7861/clinmed.2019-0218

\section{Introduction}

Faster diagnosis through accelerated pathways is a central component to the National Cancer Programme. ${ }^{1}$ NHS England are

Author: ${ }^{\text {A }}$ consultant chest physician, Wythenshawe Hospital, Manchester, UK; B lung cancer matron, Wythenshawe Hospital,

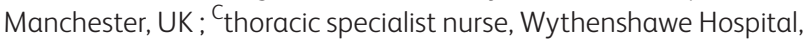
Manchester, UK; ' thoracic radiologist, Wythenshawe Hospital, Manchester, UK introducing a 'faster diagnosis standard' to ensure any patient with a suspicion of cancer completes their diagnostic tests to confirm or refute this suspicion within 28 days of referral. All cancer services will need to be compliant with this standard by April $2020 .^{2}$ The current national cancer waiting targets dictate that a patient referred with suspected cancer should see a specialist within 14 days of referral. ${ }^{3}$ This leaves little time within the pathway for further diagnostic tests and results within the current and future cancer targets.

Lung cancer is the single biggest cause of cancer death in the UK accounting for $21 \%$ of all cancer deaths. ${ }^{4}$ Data from a multicentre randomised controlled trial, in the UK, of differing lung cancer diagnostic pathways revealed a significant improvement in median overall survival (from 312 days to 503 days) by reducing the diagnostic pathway from an average of 30 days to 14 days. ${ }^{5}$ This time saving equates to the same amount as the national cancer target for first being seen by a specialist following a suspected cancer referral. Therefore, there is significant potential to improve outcomes by radically reinventing the front end of the lung cancer pathway and ensuring rapid access to diagnostic specialists.

We describe the implementation and impact of the Rapid Access to Pulmonary Investigation and Diagnosis (RAPID) programme at Wythenshawe Hospital in Manchester; a new and ambitious accelerated lung cancer pathway specifically targeting the front end of the pathway, launched in 2016.

\section{Methods}

The central ethos of the RAPID programme is to offer next day computed tomography (CT) following suspected lung cancer referral along with immediate 'hot' reporting of the CT by a specialist thoracic radiologist. The CT report coupled with a clinical assessment then informs a same day triage and management plan from a specialist respiratory physician with all the results and information provided to the patient.

Prior to implementation, an investigation into the existing processes in our radiology department revealed there was frequently a slow start to the day across the hospital's three CT machines with variable staff arrival times. An average of 1.6 scans per hour were performed between the hours of 8-9am compared to an average of 5.7 scans per hour from $9 \mathrm{am}-5 \mathrm{pm}$. Suspected lung cancer CTs were performed within generic cancer pathway 
Fig 1. Radiology vetting process for type of computed tomography performed in the RAPID programme. $\mathrm{CT}=$ computed tomography; CXR = chest $\mathrm{X}$-ray; $\mathrm{GI}=$ gastrointestinal.

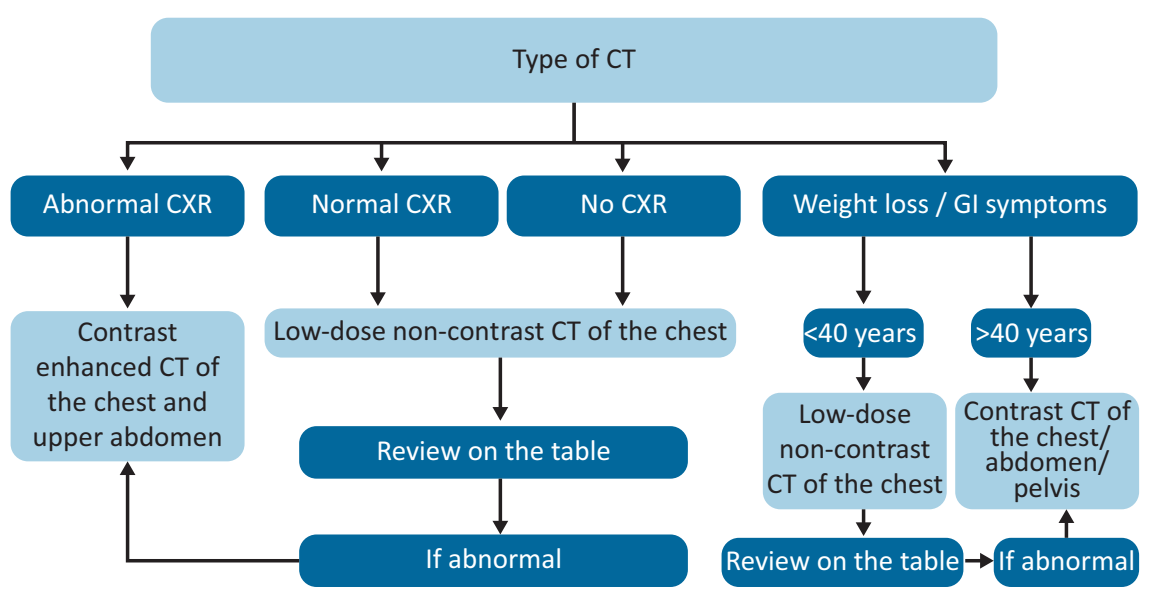

slots, in routine outpatient (OP) slots or on an ad hoc basis. Suspected lung cancer $C T$ requests were vetted remotely and the default scan was a contrast enhanced CT of the thorax and upper abdomen necessitating the provision of recent renal function. Once completed, these scans were then placed into an urgent list for reporting by the next available thoracic radiologist at an unknown time point.

An investigation into the referral pathway for general practitioner (GP) suspected lung cancer referrals revealed a number of inefficiencies. The referral needed to travel from a GP to a booking centre, to hospital cancer services, to respiratory consultant secretaries and to respiratory consultants who would then request $\mathrm{CT}$. The booking centre independently made a clinic appointment aiming to be within the 14 -day target but not knowing whether the CT would have been performed or reported by the time of this clinic appointment.

In order to achieve the ambitions of the RAPID programme, $\mathrm{a}$ number of interventions were undertaken. A pathway navigator was recruited to oversee the referral process and be the link between radiology, respiratory, booking centre and patient. A new process was implemented whereby the booking centre would contact the patient by phone on receipt of a referral and invite the patient to the CT department at 8am the following working day before the referral and patient details are handed over to the RAPID navigator. The navigator is then able to add the patient to the appointments system for both CT and outpatient clinic. Chest X-rays (CXRs) reported as suspicious for lung cancer are automatically emailed to the RAPID navigator and the same process is initiated except the navigator contacts the patient by phone inviting them for $\mathrm{CT}$ at $8 \mathrm{am}$ the following morning. Radiographer job plans were revised such that one radiographer would always start at 7.45am and have the $\mathrm{CT}$ machines ready to commence imaging at $8 \mathrm{am}$ and the spare capacity between 8-9am was utilised to ring-fence five CT appointments for the RAPID programme, Monday to Friday. A point-of-care renal profile machine (finger prick) was purchased and calibrated to allow immediate renal function results where needed. Radiologist job plans were amended to provide consultant radiologist presence throughout the CT period of 8-9am. To ensure highquality radiology-led vetting of CTs a number of processes were implemented to ensure the radiologists had access to all previous imaging across the Greater Manchester region (regional picture archiving and communication system (PACS)) and the GP referrals (uploaded electronically by the RAPID navigator). This allows real-time decision making on the type of CT performed (including 'on the table' review of images; Fig 1) and ensures compliance with Ionising Radiation (Medical Exposure) Regulations (IRMER). The same radiologist is then able to hot report the $C T$. The lung cancer physician job plans were amended to provide consultantled triage every morning following the hot reporting of CTs. This allowed patients to be seen immediately following the CT with a formal report and benefit from a management plan formulated in the triage process. A team of specialist nurses support the RAPID pathway through a 'meet and greet' at 8am to meet patients on arrival and explain the process for the morning, undertaking a formal patient clerking to facilitate the triage process prior to consultant review and provide nursing input into the triage process. A healthcare assistant is available for measurement of routine observations and routine bloods and electrocardiography where needed. The triage process involves a consultant lung cancer physician, a RAPID nurse and the RAPID patient navigator who utilise the information in the GP referral, the nurse-led clerking and the $\mathrm{CT}$ report to formulate a management plan before discussing the outcome with the patient. After completing a review of all patients undergoing $\mathrm{CT}$ that morning, this RAPID team can then triage any other new referrals such as radiology upgrades of suspected lung cancer (eg incidental finding on CT colonography) and complete a virtual ward round of every patient on the lung cancer pathway at that time and review any new results that may need actioning (eg positron emission tomography (PET)-CT result). This activity is co-located in a dedicated outpatient facility of four clinic rooms and a central 'hub' for the triage process.

The RAPID programme launched in April 2016 and continues to run to this day. All patients referred to the RAPID programme are prospectively recorded in a database including key dates of each stage of the pathway with pre-defined outcomes. To assess the impact of this new pathway we evaluated the following metrics: time (days) from either GP referral with suspected lung cancer or from an upgraded CXR report to RAPID CT; the proportion of RAPID CTs that were immediately hot reported; the proportion of RAPID CT reports triaged and actioned the same day by the lung cancer physician team; and the overall time (days) from referral to patient consultation with CT results. To validate these results, we compared them to data from the national Cancer Waiting 


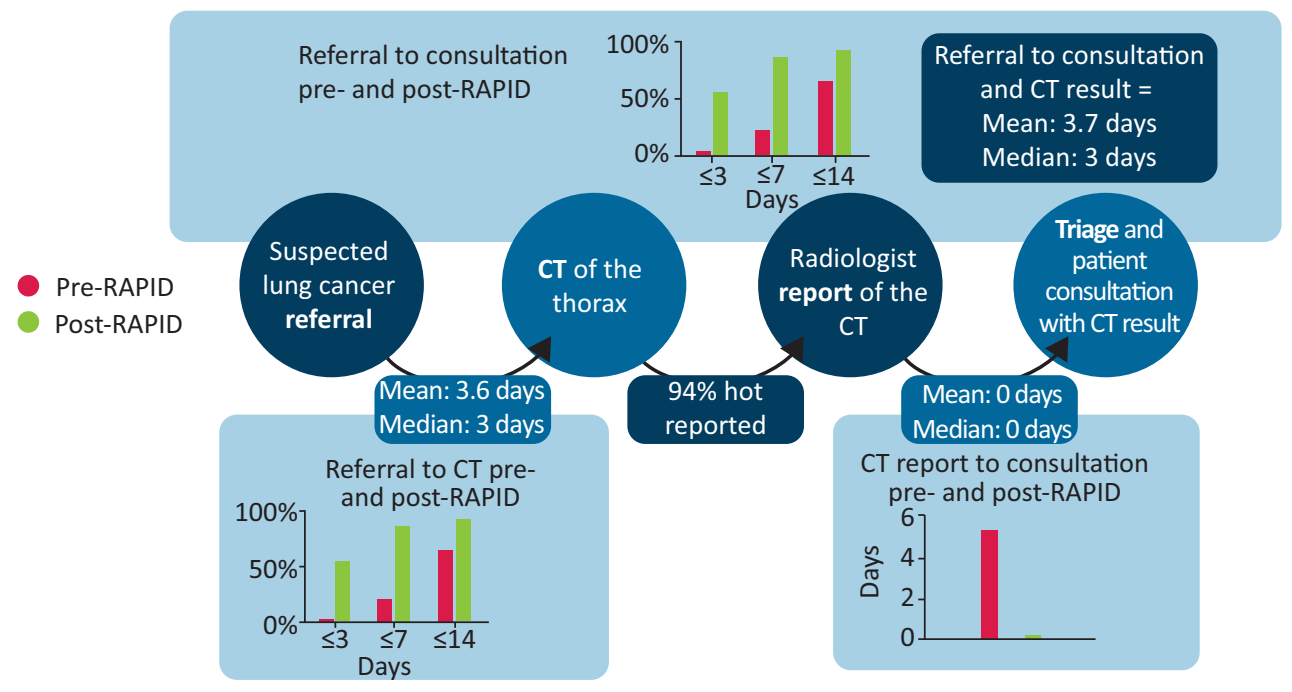

Fig 2. Overview of the RAPID programme outcomes. The RAPID pathway, 1,027 patients from April 2016 - January 2019 $\mathrm{CT}=$ computed tomography.

Times through the Greater Manchester Cancer Intelligence Team and NHS Digital. The results were also compared to pre-RAPID programme cancer performance data from Wythenshawe Hospital. Finally, to examine any potential negative impact of the RAPID programme we examined the waiting times from request to CT and CT to report for all non-RAPID CT of the thorax as well as the total number of urgent suspected lung cancer referrals from 2015 to 2018 through the hospital's radiology performance and cancer services data, respectively.

\section{Results}

From April 2016 to January 2019, 1,243 patients were referred with suspected lung cancer, either by their GP or due to an abnormal CXR, to the RAPID programme at Wythenshawe Hospital. There was missing data in 208 within the prospective database and these were excluded from this analysis, leaving 1,035 patients with full datasets. Median age was 67 years (range $57-76$ ) and $52 \%$ $(539 / 1,035)$ were male. In $0.8 \%(8 / 1,035)$ of patients, no CT was performed as the vetting radiologist felt there was no indication for a CT of the thorax based on clinical history, previous imaging and/or CXR findings.

Therefore, 1,027 patients underwent a CT of the thorax with the type of CT defined in Fig 1. The mean and median time from referral to CT was 3.6 and 3 days, respectively (Fig 2). The proportion of patients that had their CT performed within 3, 7 and 14 days from referral was $56 \%, 91 \%$ and $99 \%$, respectively. This compared with $0 \%, 27 \%$ and $74 \%$ prior to the RAPID programme. Overall, 94\% $(960 / 1,027)$ of CTs were hot reported immediately after completion. The most common reason for non-hot reporting was that patients were unable to attend at $8 \mathrm{am}$ (often more elderly and frail) and preferred a scan at a later time of day. Such patients were booked into generic cancer referral slots as per the pre-RAPID pathway without the ability for hot reporting.

The mean and median time from the CT being performed and the result being triaged/actioned by the chest physician team with the outcome discussed with the patient was 0 days (Fig 2). The proportion of patients that had their CT result triaged on the same day as the CT was $91 \%$ and within 24 hours was $95 \%$. The mean and median time from referral to consultation with a specialist was 3.7 and 3 days, respectively (Fig 2). The proportion of patients that had a consultation with a specialist within 3, 7 and 14 days from referral was $56 \%, 90 \%$ and $99 \%$, respectively (all of which had undergone CT prior to the consultation). This compared with $0 \%, 24 \%$ and $84 \%$ prior to the RAPID programme (and not all patients had undergone CT prior to consultation). Cancer Waiting Times data via NHS Digital confirmed that the median time from referral to first being seen for suspected lung cancer referrals at Wythenshawe Hospital for 2015/16, 2016/17, 2017/18 and 2018/19 were $8,4,4$ and 3 days, respectively. This data also demonstrated that the proportion of referrals first seen within 5 days of referral in same time periods was $16 \%, 71 \%, 62 \%$ and $78 \%$, respectively. From the 1,027 patients that underwent CT, 325 (32\%) were able to be discharged back to the GP without any further action; $179(17 \%)$ were referred to alternative OP services, predominantly the general respiratory OP clinic; 206 (20\%) were patients with haemoptysis and a normal CT who were offered OP autofluorescence bronchoscopy; and 272 (27\%) required an appointment with the lung cancer service for further investigation. The remaining $4 \%$ required nodule clinic surveillance or acute hospital admission. Overall, 755 patients were informed on the same day as the CT that they did not have lung cancer. Prior to the RAPID programme patients without lung cancer waited an average of 6 days from CT to being told the results.

Radiology performance data confirmed there has been no increase in turnaround times for non-RAPID CT of the chest since the programme's introduction (Fig 3). The total number of 2-week wait (2WW) of suspected lung cancer referrals has increased year-on-year from 2015 to 2018 but appears to be a steady rate without a significant change in this trend due to the RAPID programme (Fig 4).

\section{Discussion}

Through a detailed review of existing booking, radiology and respiratory medicine processes, integration of these teams and increased efficiency though re-organisation of working patterns we have been able to deliver a significantly faster pathway 


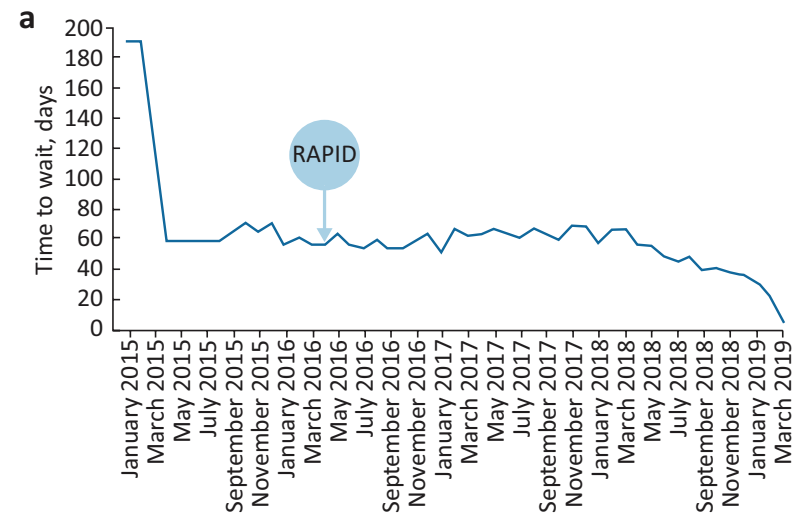

Month of request

b

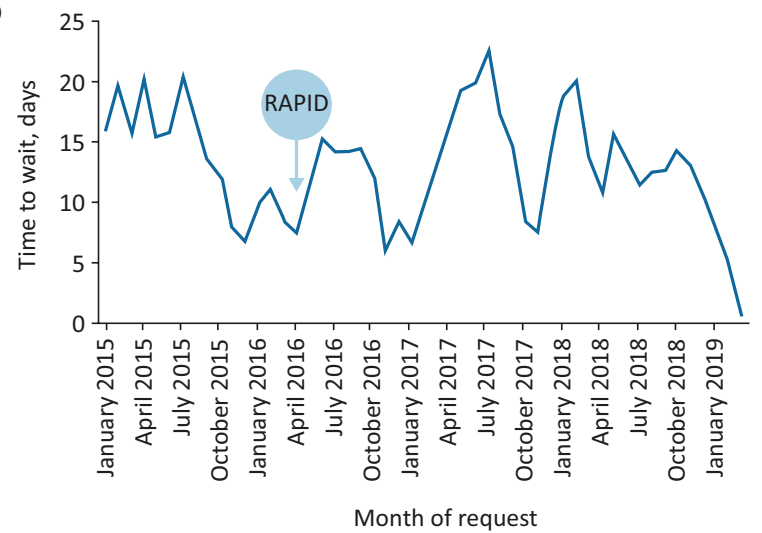

Fig 3. Average time for all non-RAPID requests of computed tomography of the thorax from 2015 to 2019. a) Time from computed tomography request to computed tomography is performed. b) Time from computed tomography is performed to reporting.

from referral to $\mathrm{CT}, \mathrm{CT}$ results and consultation with diagnostic specialists for patients with suspected lung cancer. The results are presented in calendar days for a Monday to Friday service and the results for working days would be even shorter. This improvement has been demonstrated through a prospectively collected hospital database but also validated through NHS Digital Cancer Waiting Times. The median time of 3 days from referral or CXR upgrade to

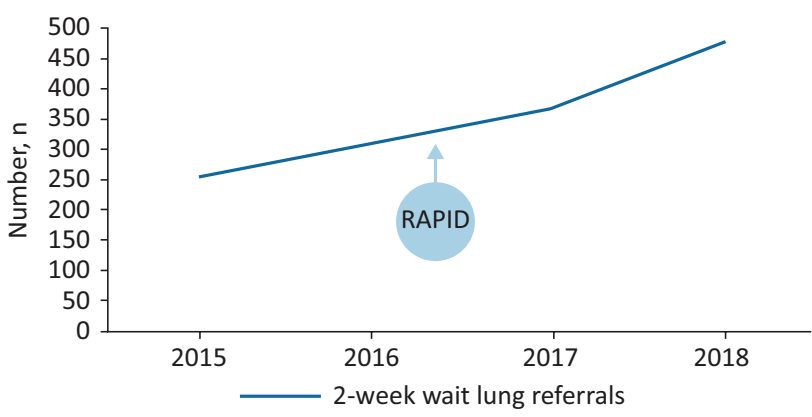

Fig 4. Volume of 2-week wait for suspected lung cancer referrals from 2015 to 2018.
$\mathrm{CT}$, triage and consultation is easily compliant with the 5 days set out in the National Lung Cancer Optimal Pathway. ${ }^{6}$ This has been achieved in the most part by re-organisation of current workload and processes plus a relatively small investment. To facilitate the implementation of the RAPID programme, a successful bid for a Health Foundation Innovation Grant was made that provided $£ 75,000$ of funding. The majority of this funding was used to backfill consultant time in job plan amendments to provide a daily service until formal job plan review and agreement was completed. A band 5 patient navigator was also funded for 2 years by Macmillan before becoming a formal permanent post. Nursing support was provided through re-organisation and expansion of the thoracic surgery nursing team. Each day, Monday to Friday, the total staffing configuration to deliver the RAPID service is as follows: 0.5 programmed activity (PA; $1 \mathrm{PA}=4$ hours) of consultant radiology time and $1 \mathrm{PA}$ of respiratory consultant time supported by two RAPID nurses, a healthcare assistant and the RAPID pathway navigator.

We have not identified any of the potential negative impacts from the RAPID programme implementation that were voiced as concerns at the time of designing the programme. We have not seen an increase in the waiting times for non-lung cancer $\mathrm{CT}$ of the thorax in either the time from request to scan or from the scan to report. The total volume of urgent suspected lung cancer referrals has steadily increased over the last 4 years and does not appear to have been influenced by the introduction of the RAPID programme (the so-called risk of 'opening the flood gates' that rapid access to $\mathrm{CT}$ might trigger). This data supports the concept that this programme is not generating any additional work but has simply focused on a re-organisation of current workload to a more efficient model. During the design of this programme, concern was also raised about the lack of chest physician vetting of referrals and decision regarding the need for CT. We have set up a comprehensive specialist thoracic radiology vetting process and, despite this, only $0.8 \%$ of patients did not require a CT. This supports the need for rapid and direct access to $\mathrm{CT}$, removing any barriers to this critical step in the pathway.

There is a building evidence base that faster pathways improves survival in lung cancer which underlines the importance of pathway redesigns such as the RAPID programme. We have already described the LungBOOST trial; a UK multicentre randomised controlled trial investigating the impact of endobronchial ultrasound (EBUS) on the diagnostic pathway in lung cancer found that those patients staged with EBUS had a shorter time to diagnosis than those that underwent conventional staging ( 14 days versus 29 days). A post-hoc analysis revealed significantly better survival in those with the shorter pathway ( 503 days versus 312 days; hazard ratio (HR) $0.60 ; 95 \%$ confidence interval (CI) $0.37-0.98 ; p=0.0382) .{ }^{5}$ Yang et al investigated the 5 -year overall survival of 4,984 patients with stage IA squamous cell carcinoma that underwent lobectomy and found that patients who had surgery 38 days or more after diagnosis had significantly worse 5-year survival than patients who had surgery earlier (HR 1.13; 95\% CI 1.02-1.25; $p=0.022$ ). ${ }^{7}$ Gomez et al examined over 28,000 Medicare claims and identified improved survival in patients with localised disease and those with distant disease that survived for greater than 1 year that waited less than 35 days from diagnosis to treatment compared to those that waited longer. ${ }^{8}$

The lung cancer pathway is complex and this paper focuses only on the front end of the pathway. For those patients with possible 
lung cancer on initial CT, an array of potential further tests awaits them including PET, brain imaging, mediastinal nodal sampling, image guided biopsies and physiological tests. ${ }^{9}$ Difficulties with capacity for these tests can induce delay into the pathway and solutions are likely to lie in high-volume diagnostic hubs. Not all hospitals can perform every test required for lung cancer staging. ${ }^{10}$ However, we firmly believe that every hospital can deliver a RAPIDlike pathway that focuses on rapid access to $C T$, hot reporting and specialist review through re-organisation of job plans and appropriate staffing, including patient navigators. There may be differences to individual pathways depending on the local resource available, for example the use of telephone consultations following CTs rather than face-to-face consultations. Setting up a RAPID pathway might also facilitate same day biopsy in patients with stage 4 disease. Such patients have the most straightforward pathway consisting only with the acquisition of adequate tissue to define the most appropriate palliative systemic anti-cancer therapy. If patients are supported appropriately following the results of their $\mathrm{CT}$, they could proceed to a biopsy procedure the same day and facilitate rapid referral to treatment. This could allow those patients with potentially curative disease that needs complex test bundles to be referred to a diagnostic hub where appropriate capacity to deliver such test bundles would need to exist.

It is also important to note the front end of the lung cancer pathway is not just dependent on CT. CXRs play a critical part of lung cancer diagnostics. It is important to place equal importance on the systems that deliver rapid CXR reporting. Therefore, on arrival to the radiology department with a primary care request for a CXR, patients at Wythenshawe Hospital are given a specific patient information (supplementary material S1) explaining that there is a chance they may require CT and this is often the next working day. They are informed a member of the hospital staff from the 'RAPID team' may contact them by telephone. An educational programme of CXR interpretation has been delivered to the hospital radiographers and instructions specifically provided to immediately alert a radiologist if a CXR appears abnormal. Every day the GP CXR list is cleared by a member of the thoracic radiology team targeting reporting within 24 hours. Any CXR reported as suspicious for lung cancer is automatically emailed to the RAPID patient navigator who will contact the patient and invite them to the CT department the following day as part of the RAPID programme.

In summary, the front end of the lung cancer pathway is often complicated by a number of inefficiencies and presents an opportunity for significant improvements and time savings. Through re-organisation of workload we have demonstrated it can be relatively straightforward to provide rapid access to CT with hot reporting and immediate triage and clinical review of patients with suspected lung cancer.

\section{Supplementary material}

Additional supplementary material may be found in the online version of this article at www.rcpjournals.org/clinmedicine:

$$
\text { S1 - Patient information provided on arrival for chest X-ray. }
$$

\section{References}

1 NHS England. Achieving world-class cancer outcomes: Taking the strategy forward. NHS, 2016. www.england.nhs.uk/wp-content/ uploads/2016/05/cancer-strategy.pdf

2 NHS England. Implementing a timed lung cancer diagnostic pathway. NHS, 2018. www.england.nhs.uk/wp-content/ uploads/2018/04/implementing-timed-lung-cancer-diagnosticpathway.pdf

3 Cancer Research UK. Cancer waiting times definitions by country. Cancer Research UK, 2015. www.cancerresearchuk.org/sites/ default/files/cs_report_cwt.pdf

4 Cancer Research UK. Lung cancer statistics. Cancer Research UK, 2016. www.cancerresearchuk.org/health-professional/cancerstatistics/statistics-by-cancer-type/lung-cancer\#heading-One

5 Navani N, Nankivell M, Lawrence DR et al. Lung cancer diagnosis and staging with endobronchial ultrasound-guided transbronchial needle aspiration compared with conventional approaches: an open-label, pragmatic, randomised controlled trial. Lancet Respir Med 2015;3:282-9.

6 NHS England Lung Clinical Expert Group. National lung cancer optimal pathway. NELCEG, 2017. http://content.smallerearthtech. co.uk/system/file_uploads/16086/original/National_Optimal_ LUNG_Pathway_Aug_2017.pdf

7 Yang $\mathrm{CJ}$, Wang $\mathrm{H}$, Kumar $\mathrm{A}$ et al. Impact of timing of lobectomy on survival for clinical stage ia lung squamous cell carcinoma. Chest 2017;152:1239-50.

8 Gomez DR, Liao KP, Swisher SG et al. Time to treatment as a quality metric in lung cancer: Staging studies, time to treatment, and patient survival. Radiother Oncol 2015;115:257-63.

9 National Institute for Health and Care Excellence. Lung cancer: diagnosis and management. NICE guideline [NG122]. NICE, 2019.

10 Cusworth K, O'Dowd E, Hubbard R et al. Variation in lung cancer resources and workload: results from the first national lung cancer organisational audit. Thorax 2015;70:1001-3.

Address for correspondence: Dr Matthew Evison, North West Lung Centre, Wythenshawe Hospital, Manchester University NHS Foundation Trust, Southmoor Road, Manchester M23 9LT, UK.

Email:m.evison@nhs.net 